\title{
ADAPTIVE BEAMFORMING USING CYCLIC SIGNALS IN THE PRESENCE OF CYCLE FREQUENCY ERROR*
}

\author{
Ju-Hong Lee ${ }^{\dagger}$ and Yung-Ting Lee \\ Room 517, 2nd Building, Department of Electrical Engineering \\ National Taiwan University Taipei, Taiwan, R.O.C.
}

\begin{abstract}
A well-known property exhibited by most manmade signals is that they generate spectral lines when they are passed through a nonlinear transform[1]. For instance, BPSK signal generates spectral lines at multiples of the symbol rate and the twice of the carrier frequency due to cyclostationary property. The Self-COherent REstoral (SCORE) algorithms presented by [2] have shown to give the optimal solution for the signal extraction problem using cyclostationary statistics. However, they suffer performance degradation in the presence of cycle frequency error. This paper considers the behavior of the SCORE algorithms in the presence of cycle frequency error. Based on the theoretical analysis, we present an efficient method to alleviate the performance degradation. Simulation result. $s$ are presented for showing the effectiveness of the proposed method.
\end{abstract}

\section{The SCORE Algorithms}

Consider an adaptive beamforming using an antenna array excited by a SOI (Signal Of Interest), $\mathrm{J}$ interferers, and background noise. If the

*This work was supported by the National Science Council Under Grant NSC85-2213-E002-008.

${ }^{\dagger}$ Author for All Correspondence. inverse bandwidth of the receiver is small with respect to the electrical distance between the array elements, then the received data vector $\mathrm{x}(t)$ is given by

$$
\begin{aligned}
\mathbf{x}(t) & =s(t) \mathbf{S}_{\mathbf{d}}+\sum_{j=1}^{J} s_{j}(t) \mathbf{S}_{\mathbf{j}}+\mathbf{n}(t) \\
& =s(t) \mathbf{S}_{\mathbf{d}}+\mathbf{i}(t)
\end{aligned}
$$

where $S_{d}$ and $S_{j}$ denote the SOI and interference aperture vectors, respectively. Assume that $s(t)$ is self-coherent at $\alpha$ which is one of the cycle frequencies of $s(t)$, and that $\mathrm{i}(t)$ is not self-coherent at $\alpha$ and is temporally uncorrelated with $s(t)$. The array output is given by

$$
y(t)=\mathbf{W}^{H} \mathbf{x}(t)
$$

From (1), we note that the extraction of the SOI can be accomplished by constructing a suitable cost function on $y(t)$ and optimizing it with respect to the weight vector $\mathbf{W}$. In the LS-SCORE algorithm, the following cost function is used[2]

$$
F_{s c}(\mathbf{W} ; \mathbf{c})=<|y(t)-r(t)|^{2}>_{T}
$$

where the reference signal $r(t)$ is given by

$$
r(t)=\mathrm{c}^{H} \mathrm{x}^{(*)}(t-\tau) \mathrm{e}^{j 2 \pi \alpha t}
$$

and $\langle\cdot\rangle_{T}$ denotes the time average over $[0, T]$. The solution of minimizing ( 3 ) is given by

$$
\mathbf{W}_{s c}=\hat{\mathbf{R}}_{x x}^{-1} \hat{\mathbf{R}}_{x r}(\alpha)
$$


where $\hat{\mathbf{R}}_{x x}$ and $\hat{\mathbf{R}}_{x r}(\alpha)$ are the sample autocor- From the property of Fourier transform, the relation matrix of $\mathbf{x}(t)$ and the cross-correlation sample cross-correlation vector can be representvector of $\mathrm{x}(t)$ and $r(t)$ computed over $[0, T]$, re- ed by spectively. It is shown in [2] that (5) converges to the maximum-SINR solution when $T$ approaches infinite for any vector of $\mathrm{c}$ as long as $\mathrm{c}^{H} \mathrm{~S}_{\mathrm{d}} \neq 0$. where $\otimes$ denotes the convolution operation and Due to the fact that $r(t)$ contains the interfer- $S I N C(f T)=\frac{\sin (\pi f T)}{(\pi f T)}$. Moreover, due to the ence, the LS-SCORE algorithm converges slow- fact that cycle frequencies are discrete, the cyclic ly. To alleviate this difficulty, Gardner [2] also autocorrelation function of the SOI can be writproposed to find the optimal weight vector $\mathbf{W}_{\text {opt }}$ ten as

and the optimal control vector $\mathbf{c}_{\text {opt }}$ by maximizing the correlation coefficient between $y(t)$ and $r(t)$

$$
\tilde{F}_{s c}(\mathbf{W} ; \mathbf{c})=\frac{\mathbf{W}^{H} \hat{\mathbf{R}}_{x u} \mathbf{c}}{\left(\mathbf{W}^{H} \hat{\mathbf{R}}_{x x} \mathbf{W}\right)\left(\mathbf{c}^{H} \hat{\mathbf{R}}_{u \psi} \mathbf{c}\right)}
$$

where $\mathbf{u}(t)=\mathrm{x}^{(*)}(t-\tau) \mathrm{e}^{j 2 \pi \alpha t}$ is the control sig nal. The solutions of maximizing (6) are given by

$$
\begin{aligned}
& \mathbf{W}_{o p t} \propto \hat{\mathbf{R}}_{x x}^{-1} \hat{\mathbf{R}}_{x u} \mathbf{c}_{o p t}=\hat{\mathbf{R}}_{x x}^{-1} \dot{\overline{\mathbf{R}}}_{x r} \\
& \mathbf{c}_{\text {opt }} \propto \hat{\mathbf{R}}_{u u}^{-1} \hat{\mathbf{R}}_{u x} \mathbf{W}_{\text {opt }}=\hat{\mathbf{R}}_{u u}^{-1} \dot{\hat{\mathbf{R}}}_{u y}
\end{aligned}
$$

$$
R_{s}^{f}(\tau)=\sum_{n} d_{n}(\tau) \delta\left(f-\alpha_{n}\right)
$$

From (8), the cross-correlation vector can be expressed by

$$
\begin{aligned}
\mathbf{R}_{x r}(f) & =\mathbf{R}_{x x}^{f} \mathrm{ce}^{-j \pi f r} \\
& =k_{d} R_{s}^{f} \mathbf{S}_{\mathbf{d}}+\sum_{j=1}^{J} k_{j} R_{s_{j}}^{f} \mathbf{S}_{\mathbf{j}}+\mathbf{k}_{n}
\end{aligned}
$$

where $k_{d}=S_{d}{ }^{H} c e^{-j \pi f \tau}, k_{j}=S_{j}{ }^{H} c e^{-j \pi f \tau}$ and $\mathbf{k}_{\mathbf{n}}=\mathbf{R}_{\mathbf{n}}^{\mathbf{f}}(\tau) \mathrm{ce}^{-\mathrm{j} \pi \mathrm{f} \tau}$. Substituting (12) into (10) (7) yields (Actually, $\hat{\mathbf{R}}_{x r}(f)$ includes the cyclic cross correlations between the SOI, interference, and where $\hat{\tilde{\mathbf{R}}}_{x r}=\hat{\mathbf{R}}_{x u} \mathbf{c}_{o p t}$ and $\hat{\tilde{\mathbf{R}}}_{x y}=\hat{\mathbf{R}}_{u x} \mathbf{W}_{o p t}$. It is noise. However, they are negligible due to large shown[2] that $\mathbf{W}_{\text {opt }}$ also maximizes the output $T$.)

SINR as $T \rightarrow \infty$. Simulation results show that the performance of Cross-SCORE is much better than LS-SCORE, especially in the presence of strong interference.

\section{Performance Analysis}

From (1), we obtain (by letting $f=\alpha$ )

$$
\begin{aligned}
\mathbf{R}_{x x}^{f} & =\left\langle\mathbf{x}\left(t+\frac{\tau}{2}\right) \mathbf{x}^{H}\left(t-\frac{\tau}{2}\right) e^{-j 2 \pi f t}\right\rangle_{\infty} \\
& =R_{s}^{f} \mathbf{S}_{\mathbf{d}} \mathbf{S}_{\mathbf{d}}{ }^{H}+\sum_{j=1}^{J} R_{s j}^{f} \mathbf{S}_{\mathbf{j}} \mathbf{S}_{\mathbf{j}}{ }^{H}+\mathbf{R}_{n}^{f}
\end{aligned}
$$

where

$$
\begin{aligned}
\hat{\mathbf{R}}_{x r}(f) & =k_{d}\left\{\sum_{n} d_{n}(\tau) S I N C\left(\left(f-\alpha_{n}\right) T\right)\right\} \mathbf{S}_{\mathbf{d}} \\
& +\sum_{j, m} k_{j}\left\{\rho_{j, m}(\tau) S I N C\left(\left(f-\beta_{j, m}\right) T\right)\right\} \mathbf{S}_{\mathbf{j}} \\
& +\tilde{\mathbf{k}}_{n n} \otimes S I N C(f T)
\end{aligned}
$$

where non-cyclostationary interference and noise are contained in $\tilde{\mathbf{k}}_{n n}$. Without loss of generality, we set the known cycle frequency $\alpha=\alpha_{1}$. If $\alpha$ and $\beta_{j, m}$ are well separated and due to the fact that the value of $S I N C(f T)$ is small for large

(8) $f T$ then the effect of interference and noise in (13) is small. When $f=\alpha+\Delta \alpha$, where $\Delta \alpha$ is the amount of deviation, (13) can be written by

$$
R_{a}^{s}=<a\left(t+\frac{\tau}{2}\right) a^{*}\left(t-\frac{\tau}{2}\right) e^{-j 2 \pi f t}>_{\infty}
$$

(9) $\hat{\mathbf{R}}_{x r}(f)=k_{d}\left\{d_{1}(\tau) S I N C(\triangle \alpha T)\right.$ 
$\left.+\sum_{n \neq 1} d_{n}(\tau) S I N C\left(\tilde{\alpha}_{n} T\right)\right\} \mathbf{S}_{\mathbf{d}}$
$+\sum_{j, m} k_{j}\left\{\rho_{j, m}(\tau) S I N C\left(\tilde{\beta}_{j, m} T\right)\right\} \mathbf{S}_{\mathbf{j}}$
$+\tilde{\mathbf{k}}_{n} \otimes S I N C((\alpha+\Delta \alpha) T)$

cross-correlation vector when $f=\alpha+\Delta \alpha$ is also given by

$\hat{\mathbf{R}}_{x \dot{r}}(f)=k_{d}\left\{d_{1}(\tau) \Omega(\Delta \alpha T)\right.$

$\left.+\sum d_{n}(\tau) \Omega\left(\tilde{\alpha}_{n} T\right)\right\} S_{\mathbf{d}}$

$+\sum_{j, m} k_{j}\left\{\rho_{j, m}(\tau) \Omega\left(\tilde{\beta}_{j, m} T\right)\right\} \mathbf{S}_{\mathbf{j}}$

$+\tilde{\mathbf{k}}_{n} \otimes \Omega((\alpha+\Delta \alpha) T)$

$\triangle \alpha . \quad$ Due to the fact that $\operatorname{SINC}(\triangle \alpha T)=0$ when $T=T_{n}=\frac{ \pm n}{\Delta \alpha}, n=1,2, \ldots, \hat{\mathbf{R}}_{x \tau}(f) \cong$ $k_{d} d_{1}(\tau) S I N C(\triangle \alpha T) \mathbf{S}_{\mathbf{d}}$ as $T$ is far from $T_{n}$. However, when $T$ is near to $T_{n}$, the components of $S_{\mathbf{j}}$ in (14) are not negligible and thus the performance of the SCORE algorithms becomes poor. From (7), we note that the difference between LS-SCORE and Cross-SCORE is that Cross-SCORE adjusts adaptively the control vector $\mathrm{c}$ and thus the behavior of the CrossSCORE algorithm is similar to the LS-SCORE algorithm when cycle frequency is error.

\section{An Efficient Method}

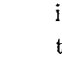

Consider the following new reference signal in the LS-SCORE algorithm

$\tilde{r}(t)=\{\gamma+S I N C(\kappa t)\} \mathrm{c}^{H} \mathbf{x}^{(*)}(t-\tau) e^{j 2 \pi \alpha t}$

where both $\gamma$ and $\kappa$ are two constants. Accordingly, we also obtain the new control signal $\tilde{u}(t)=\{\gamma+S I N C(\kappa t)\} \mathrm{x}^{(*)}(t-\tau) e^{j 2 \pi \alpha t}$ in the Cross-SCORE algorithm. From (15), we obtain

$$
\begin{aligned}
\mathbf{R}_{x \bar{r}}(f) & =\gamma \mathbf{R}_{x \tau}(f)+\frac{1}{\kappa} \operatorname{rect}\left(\frac{f}{\kappa}\right) \otimes \mathbf{R}_{x r}(f) \\
& =k_{d}\left(\gamma R_{s}^{f}+\frac{1}{\kappa} \operatorname{rect}\left(\frac{f}{\kappa}\right) \otimes R_{s}^{f}\right) \mathbf{S}_{\mathbf{d}} \\
& +\sum_{j=1}^{J} k_{j}\left(\gamma R_{s_{j}}^{f}+\frac{1}{\kappa} \operatorname{rect}\left(\frac{f}{\kappa}\right) \otimes R_{s j}^{f}\right) \mathbf{S}_{\mathbf{j}} \\
& +\left(\gamma \mathbf{k}_{n}+\frac{1}{\kappa} \operatorname{rect}\left(\frac{f}{\kappa}\right) \otimes \mathbf{k}_{j_{k}}\right.
\end{aligned}
$$

where $\Omega(f T)=\gamma S I N C(f T)+\frac{1}{\kappa} \operatorname{rect}\left(\frac{f}{\alpha}\right) \otimes$ $S I N C(f T)$. Under the conditions that $\triangle \alpha=$ 0 and $\triangle \alpha \neq 0, \mathbf{R}_{x \dot{r}}(f)$ is proportional to $\mathbf{S}_{\mathbf{d}}$. The result is due to the fact that from $(16), \operatorname{rect}\left(\frac{f}{\kappa}\right) \otimes R_{s}^{f}=\sum d_{n}(\tau) \operatorname{rect}\left(\frac{f-\alpha_{n}}{\kappa}\right)$ is not equal to 0 as long as $\Delta \alpha \leq \frac{\kappa}{2}$. Moreover, owing to $\operatorname{rect}\left(\frac{\Delta \alpha}{\kappa}\right) \otimes S I N C(\triangle \alpha T)$ and $S I N C(\triangle \alpha T)$ in (17) are not equal to 0 at the same time, $\hat{\mathbf{R}}_{x i}$ is not seriously effected by $\mathbf{S}_{\mathbf{j}}$ when $T$ is near to $T_{n}=\frac{ \pm n}{\Delta \alpha}$. As a result, using $\tilde{r}(t)$ in the LSSCORE algorithm and $\tilde{u}(t)$ in the Cross-SCORE algorithm make these algorithms robust against the effect of cycle frequency error. We note that $\operatorname{rect}\left(\frac{f}{\kappa}\right) \otimes S I N C\left(\tilde{\beta}_{j, m} T\right)$ in $(17)$ enhances the components of $\mathbf{S}_{\mathbf{j}}$ in $\hat{\mathbf{R}}_{x \dot{r}}(f)$ and leads to performance degradation. However, choosing larger $\gamma$ can alleviate this difficulty. Furthermore, the components of $\mathbf{S}_{\mathbf{j}}$ in (17) become larger as $\kappa$ or $\gamma$ increases. Therefore, by choosing the values of $\gamma$ and $\kappa$ adequately, we can alleviate the effect of cycle frequency error on the performance of the SCORE algorithms.

\section{Simulation and Observation}

Consider an adaptive beamformer using a $21 \mathrm{u}$ niform linear array with interelement spacing $=$ (16) half wavelength of the desired signal. All of the inputs are BPSK. The carrier frequency of the where $\operatorname{rect}\left(\frac{f}{\kappa}\right)$ equals 1 when $-\frac{\kappa}{2} \leq f \leq \frac{\kappa}{2}$ and desired signal is set to 1 . The noise is spatialequals 0 , elsewhere. The corresponding sample ly white. Moreover, we set $\gamma=0.3, \kappa=0.02$, 
and the sampling interval $T_{s}=0.2$. Two cases in which $J$ equals 0 and 2 are considered. For each case, $f=2$ and $f=2.01$ (i.e., cycle frequency error $=0.01$ ) are used. The vector $c$ in the LS-SCORE algorithm is fixed and given by $c=[1,0,0, \ldots, 0]^{T}$. From (14), the coefficient of $\mathrm{S}_{\mathrm{d}}$ is proportional to $S I N C(\triangle \alpha T)$ when $J=0$. Owing to $S I N C(\triangle \alpha T)=0$ when $T=\frac{ \pm n}{\Delta \alpha}$, $n=1,2, \ldots$, there are nulls at about $500 n$ snapshots which are shown in Figure 1(b) when using the LS-SCORE and Cross-SCORE algorithms. In the case of $J=2$, nulls still appear at about 500 n snapshots which are shown in Figure 2(b). For both cases, the proposed method improves the performance of the SCORE algorithms in the presence of cycle frequency error. Moreover, we note that an acceptable performance can be achieved by choosing appropriate $\gamma$ and $k$.

\section{References}

[1] W. A. Gardner, "Exploitation of spectral redundancy in cyclostationary signals," IEEE SP MAGAZINE, pp. 14-36, Apr. 1991.

[2] B. G. Agee, S. V. Schell and W. A. Gardner, "Spectral self-coherence restoral: A new approach to blind adaptive signal extraction using antenna arrays," Proc. IEEE, Vol. 78, pp. 753-767, Apr. 1990.

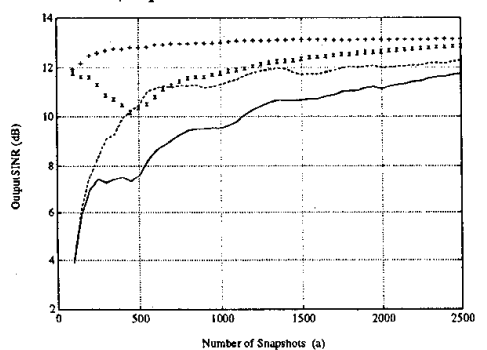

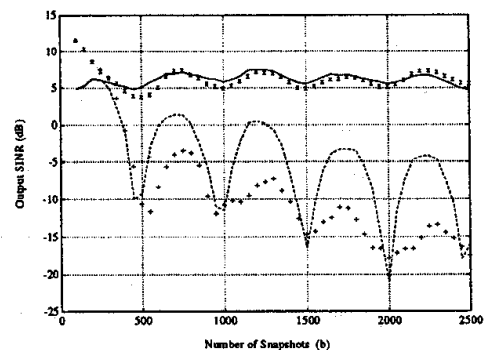

Fig. 1: $J=0$. (a): $f=2, \quad$ (b): $f=2.01$,

-' line: LS-SCORE using $\bar{r}(t)$. 'x' line: Cross-SCORE using $\bar{u}(t)$

'. - line: LS-SCORE. ' + ' line: Cross-SCORE.
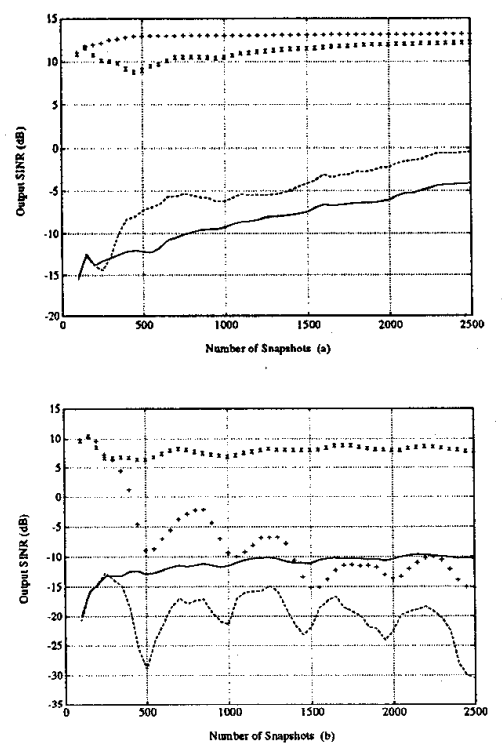

Fig. 2: $J=2$. (a): $f=2, \quad$ (b): $f=2.01$.

'-' line: LS-SCORE using $\tilde{r}(t)$. ' $x$ ' line: Cross-SCORE using $\bar{u}(t)$

'. - line: LS-SCORE. 't' line: Cross-SCORE. 